\title{
Identification of mutations associated with Macozinone-resistant in
}


AbSTRACT Macozinone is identified as a drug candidate and is currently under clinical development for the treatment of tuberculosis, but the mutations conferring resistance to Macozinone remain inadequately characterized. Herein, we investigated the Macozinone -resistance -associated mutations through selecting resistant isolates in vitro. Macozinone-resistant isolates were obtained through induction in vitro. The level of Macozinone -resistant strains was confirmed by MABA test. PCR sequencing analysis was carried out on dprE1 gene. Whole Genome Sequencing was performed to identify mutations associated with Macozinone -resistance. The totals of isolates obtained at Macozinone concentrations of $6.4 \mathrm{ng} / \mathrm{ml}$, 25.6ng/ml, 50ng/ml and 100ng/ml were 49, 20, 20 and 4 respectively. Among the 49 strains obtained by 6.4ng/ml Macozinone only one strain had C387S mutation in dprE1. C387S is only occurred in high-level resistant isolates (MIC > 500ng/ml). Meanwhile high-level resistance to Macozinone can occur in strains induced at $6.4 \mathrm{ng} / \mathrm{ml}$ and the frequency of occurrence is low $(1 / 49,2.04 \%)$. The $\mathrm{MIC}_{90}$ of other strains except the strains carrying C387S mutation is at the same level $\left(11.5 \mathrm{ng} / \mathrm{ml}>\mathrm{MIC}_{90}>2 \mathrm{ng} / \mathrm{ml}\right)$. The G61A or G248A mutations in dprE1 was discovered for the first time. Other gene mutations (rv0678, rrs, mbtF, rv2956, et al) were found in low-level resistant strains.

Conclusions: High-level resistant isolates can be produced at low concentration of Macozinone. C387S mutation in dprE1 is directly related to high-level resistance. There may be new mechanisms involved in Macozinone-resistance independent of dprE1 mutations.

KEYWORDS Mycobacterium tuberculosis, DprE1, Macozinone, drug resistance mechanisms, Whole genome sequencing 
Tuberculosis (TB) caused by Mycobacterium tuberculosis (M.tb) is an infectious disease that affects more than 10 million people worldwide every year, mainly in developing countries (1). It is estimated that approximately 1.7 billion people are infected with M.tb (1). Moreover, M.tb is still a global leading cause of death (1). The emergence and widespread of drug-resistant TB is now recognized as one of the most dangerous threats to global TB control (2). Patients with multidrug-resistant TB (MDR-TB), extensively drug-resistant TB (XDR-TB), and totally drug-resistant TB (TDR-TB) need long and expensive treatment (3-5). WHO approved anti-tuberculosis therapies can only treat less than 50\% of MDR-TB and 30\% of XDR-TB $(6,7)$. There is an urgent need for the development of more efficient new drugs and shorter treatment regimens.

Macozinone (MCZ), previously known as PBTZ169, is currently undergoing Phase $1 / 2$ clinical trails for the treatment of tuberculosis $(8,9)$. MCZ had good bactericidal activity against multidrug-resistant tuberculosis strains and was effective against $M . t b$ in replication phase (10-12). In the mouse infection model, MCZ showed excellent therapeutic effect. Other studies have also found that MCZ has synergistic effect with bedaquiline (10). MCZ targets the essential flavoenzyme DprE1 and blocks the synthesis of the cell wall precursordecaprenyl-phospho-arabinose (DPA) and provoking lysis of M.tb (10,13-19). Recent studies have confirmed that MCZ can form stable covalent complexes with DprE1 by targeting DprE1 enzyme (20). DprE1 plays a catalytic role together with DprE2 and catalyzes the epimerisation of decaprenyl-phospho-ribose (DPR) to DPA. DPA is the only donor of D-arabinose in mycobacteria (16-20). Arabinose polymers form DPA, which is one of the cell wall components of mycobacteria. The inhibition of MCZ on DprE1 eventually led to cell lysis and cell death (17). 
It has also been verified that Cys387 in dprE1 is essential for the activity of DprE1 inhibitors (11). Cys387 plays an important role in covalent binding with MCZ and drug resistance occurs when the site is substituted by other amino acids $(10,17,18)$. However, It is not clear whether the substitution of Cys387 residue in DprE1 is the single reason of MCZ-resistance. The characterization of MCZ-resistance is still unclear. In this study, the occurrence of resistance to MCZ similar to that of drug-resistant $\mathrm{TB}$ in clinical therapy was obtained through rising gradually $\mathrm{MCZ}$ concentration induction in vitro, so as to find out the mutations of drug resistance gene and to better understand the mechanisms of MCZ-resistance, which would be helpful for monitoring the resistance to $\mathrm{MCZ}$ and developing more accurate molecular tests in future clinical application.

\section{RESULTS}

\section{Isolation of MCZ -induced isolates and Determination of the minimum}

inhibitory concentration (MIC). The $\mathrm{MIC}_{90}$ of $\mathrm{MCZ}$ for M.tb $\mathrm{H} 37 \mathrm{Rv}$ determined by MABA was $0.2 \mathrm{ng} / \mathrm{ml}$. To isolate induced MCZ-resistant strains, early stationary phase cultures of $\mathrm{H} 37 \mathrm{Rv}$ were plated on $7 \mathrm{H} 10$ agar plates containing $0.1 \mathrm{ng} / \mathrm{mL} \mathrm{MCZ}$. Isolated strains were obtained through several rounds of selection. After repeated MCZ susceptibility testing to rule out false resistance, 4 isolated strains obtained by MCZ concentration of $100 \mathrm{ng} / \mathrm{ml}, 20$ isolated strains obtained by MCZ concentration of 50ng/ml, 20 isolated strains obtained by MCZ concentration of $25.6 \mathrm{ng} / \mathrm{ml}$ and 49 induced strains obtained by MCZ concentration of $6.4 \mathrm{ng} / \mathrm{ml}$ were selected. Only 4 strains survived when the MCZ concentration in the $7 \mathrm{H} 10$ medium increased to 100ng/ml. The $\mathrm{MIC}_{90}$ values of above isolated strains were measured respectively (shown in table 1). Because there is no clinical MCZ-resistance break point now, 
MIC90 value of isolates higher than $2 \mathrm{ng} / \mathrm{ml}$ is defined as $\mathrm{MCZ}$ resistance $(>10 \times$ MIC90 of $\mathrm{H} 37 \mathrm{Rv}$ ), and $\mathrm{MIC} 90$ value higher than $500 \mathrm{ng} / \mathrm{ml}$ is defined as $\mathrm{MCZ}$ high-level resistance in our study. Finally, a total of 4 high-level resistant strains were obtained, among which 3 strains (S1-1,S2-1,S3-1) isolated from the medium with MCZ concentration of $100 \mathrm{ng} / \mathrm{ml}$ and 1 strain (S4-4) isolated from the medium with MCZ concentration of $6.4 \mathrm{ng} / \mathrm{ml}$. The emergence of S4-4 showed that high-level resistance to $\mathrm{MCZ}$ can occur in stains induced at low-level drug concentration. Meanwhile by the induction of $6.4 \mathrm{ng} / \mathrm{ml} \mathrm{MCZ}$, the frequency of occurrence of high-level resistance (1/49) is low which showed that it is difficult to produce high-level resistance strains to MCZ. Although strains S4-1, S4-2 and S4-3 were also obtained by culture step by step, they were thought to be the same strain because of their same genome sequence by sequencing analysis of the whole genome.

Except for 4 high-level resistant strains, the rest of isolated strains had lower and similar $\mathrm{MIC}_{90}$ value $\quad(11.5 \mathrm{ng} / \mathrm{ml}>\mathrm{MIC} 90>2 \mathrm{ng} / \mathrm{ml})$, which were more than 10-fold MIC of Wide-type H37Rv. For these low-level MCZ-resistant strains, even the concentration of $\mathrm{MCZ}$ in $7 \mathrm{H} 10$ solid medium rised to $50 \mathrm{ng} / \mathrm{ml}$, MIC90 were no longer increased (Table 1).

dprE1 sequencing. We isolated genomic DNA from the $49 \mathrm{MCZ}$-induced strains at the concentration of $6.4 \mathrm{ng} / \mathrm{ml}$ and performed PCR to amplify 674 bp DNA fragment of dprE1 gene. Only strain S4-4 had C387S mutation (tGc/tCc, 1/49, 2.04\%) whose MIC was $>500 \mathrm{ng} / \mathrm{ml}$, whereas other 48 strains had no C387S mutation whose MIC was between $2 \mathrm{ng} / \mathrm{ml}$ and $10.3 \mathrm{ng} . \mathrm{ml}$. This result suggested that there is an obvious correlation between C387S mutation in dprE1 and MCZ-resistance level. In order to know whether the mutation of C387S may occur under the lower MCZ concentration, Cys387 in S4-5 obtained by inducing at MCZ concentration of $3.2 \mathrm{ng} / \mathrm{ml}$ had also 
been detected. We found that S4-5 had no C387S mutation. Considering C387S mutation can occur in strain $\mathrm{S} 4-4$ at the concentration of $6.4 \mathrm{ng} / \mathrm{ml}$, this result means low concentration MCZ induction can produce high-level drug-resistance and C387S mutation in dprE1 only occurs in high-level MCZ-resistant strains.

\section{Sequencing Analysis of the Whole Genome of H37Rv MCZ-resistant Strain. To} explore the development of MCZ resistance in vitro and to compare the genes changes before and after drug induction, a total of $9 \mathrm{MCZ}-$ resistant strains were subjected to whole genome sequencing using Illumina HiSeq platform. The 9 strains were S2-1, S2-2, S2-4, S3-1, S3-2, S3-4, S4-1, S4-4 and S4-5. Among these isolates S2-1, S3-1, S4-1, S4-4 were high-level resistance strains (MIC > 500ng/ml), whereas others were low-level resistant strains (MIC > 2ng/ml). We found that S4-1 and S4-4 have C387S mutation $(\mathrm{tGc} / \mathrm{tCc})$ which is consistent with the previous PCR results. Another 2 high-level resistant strains (S2-1, S3-1) also had C387S mutation (Tgc/Agc, tGc/tCc). It is interesting to note that 3 strains (S2-1, S2-2 and S2-4) had G248A mutation in the dprE1 gene (gGc/gCc) and 3 strains (S3-1,S3-2 and S3-4) had G61A mutation in dprE1 (gGg/gCg).

Comparative genome sequence analyses of the 9 strains revealed that they all had missense mutations in 3 genes, fadD2 (R137P, 9/9, 100\%), phoT (Q153R, 9/9, 100\%) and rv1725c (E28G, 9/9, 100\%); they also had frame shift mutation in SenX3 gene (animo acid 330 site, C/CG, 9/9, 100\%) (Table 2). Isolates S2-1, S2-2 and S2-4 also had A99V substitution in rv0678 (3/9, 30\%), Q1026H substitution in $\operatorname{rrs}(3 / 9,30 \%)$ and A108T substitution in rv2956 (3/9, 30\%). A270G substitution in mbtF appears in isolates S2-1, S2-2, S2-4, S4-1, S4-4 and S4-5 (6/9, 66.7\%). Isolates S3-1 had a S290P mutation in rv3230c $(1 / 9,11.1 \%)$. Intergenic region of rv1706A-rv1706c mutation was only found in S3-1 and S3-2 (2/9, 22.2\%). S4-1 and S4-4 had also 
identical aofH mutation G383S $(2 / 9,22.2 \%)$. Our findings suggested that these mutations independent of dprE1 may be related to the increased sensitivity of $\mathrm{MCZ}$ induced low-level resistant strains.

\section{DISCUSSION}

MCZ is a bactericidal benzothiazinone that inhibits the essential flavoprotein DprE1 by forming acovalent bond with the active-site Cys387 residue, thus preventing the synthesis of decaprenyl-phosphoryl-ribose. MCZ resistance in M.tb is caused by mutations in dprE1 gene. A previous study that employed genetic approaches confirmed that rv3790 gene in BTZs-resistant $M$. $t b$ mediated increased resistance and mutants harbor missense mutations in this gene (14). DprE1(Rv3790) is a drug target of MCZ $(8,14,15,17)$. It had been verified that Cys387 is essential for the activity of DprE1 inhibitors by method of promiscuous site-directed mutagenesis to introduce other codons at Cys387 into dprE1 of M.tb (18). The Cys387 residue of DprE1 is highly conserved in orthologous enzymes in mycobactria and substitution of Cys387 with alanine or serine in M.avium, M.intracellulare and M.abscessus may weaken the binding affinity between the drug and the target, thus showing the natural drug resistance of these NTM strains to $\operatorname{MCZ}(13,18,19)$.

In this study we obtained M.tb MCZ-resistant isolates in vitro through $\mathrm{H} 37 \mathrm{Rv}$ growing on agar plates containing $\mathrm{MCZ}$ from low concentration to high concentration. We found that C387S mutation in dprE1 only occurred in high-level MCZ resistant isolates which is consistent with previous report in which Cys387 position harboring alleles showed high MCZ resistance (18). Drug resistance occurs spontaneously in $M . t b$ at a different rate for each drug. For example, mutations resulting in resistance to RIF occur at a rate of $10^{-10}$ cell division, compared with $10^{-7}-10^{-9}$ for INH (21). The 
frequency of Cys387 amino acid position in dprE1 harboring alleles is middle $\left(<10^{-8}\right)$ (8). This kind of middle spontaneous mutation rate $\left(<10^{-8}\right)$ may explain the emergence of high-level resistant strains to MCZ by the induction at low drug concentration of $6.4 \mathrm{ng} / \mathrm{ml}$ in our research. This phenomenon also means that it may be one-step mutation of dprE1 gene in strain S4-4, rather than the accumulation of mutations causing high-level resistance. The result also suggested that selective pressure of drug on strain is obvious and attention should be paid to the possibility of drug resistance of M.tb at low MCZ blood drug concentration caused by irregular or low-dose administration in future clinical application. Especially for the patients with lung cavities the risk of drug resistance may be higher because lung cavities frequently contain $10^{7}$ resistant bacilli emerging naturally without antimicrobial pressure, then the use of anti-mycobacterial drugs is prone to select the resistant population (22-24).

In addition, G61A and G248Amutations in dprE1were discovered for the first time. They appeared not only in high-level resistant isolates but also in low-level resistant isolates. So their contribution in the MCZ-resistance remained to be determined in the following research. We obtained a number of low-level resistant isolates whose decreased sensitivity to MCZ isn't illustrated by dprE1 mutation. Considering the mutations in known gene can't explain MCZ resistance in these isolates the possibilities must be considered that there may be other gene mutations conducive to MCZ resistance. In our research we indeed found some mutations in several genes, such as Rv0678, Rv3230c et al. But these genes mutation were detected in only 9 strains, it is difficult to speculate that they are related to low-level resistance to MCZ. More gene mutations that may be related to $\mathrm{MCZ}$ resistance would be found by expanding the sequencing number of induced isolates in future research.

$$
\text { It is confirmed that resistant bacteria is prone to fitness cost, taking the form of the }
$$


decreased viability and virulence compared to the wild-type strain in the absence of antibiotics. Fitness cost is common in bacteria (25-30). Drug-resistance of bacteria often changes some structures such as DNA helicases, ribosome, RNA polymerase, cell wall, and so on. This kind of change often leads to the decrease of bacterial growth speed and virulence, which means the acquisition of drug-resistance needs to pay an adaptive cost $(31,32)$. Therefore, the occurrence of drug-resistant mutations is a double-edged sword. Similar phenomena also exist in INH-resistant or RIF-resistant M.tb (33,34). However, drug-resistant bacteria can restore their fitness through evolution, a process called "compensatory evolution". One of the reason of compensatory evolution is that drug-resistant bacteria can further accumulate other specific mutation through evolution change. Drug-resistant M.tb also can accumulate compensatory mutations and may recovery the fitness, which is helpful for the spread of drug-resistant M.tb in the population (35-40). In our study, all of the 9 strains had nonsynonymous mutations in four non-drug-resistant related genes (fadD2, phoT, rv1725c and SenX3) changed significantly during drug induction, suggesting a possible compensatory evolutionary mechanism. On the other hand, the relationship between bacterial drug resistance and its fitness provides a new insights into analyzing the causes of drug resistance and exploring how to reduce the possibility of drug resistance.

There are limitations that must be taken into account. Firstly, there is no clinical strain yet, and the induced strains were taken as the research object; Secondly, less drug-resistant strains were obtained. The number of whole genome sequencing strains was small. However, this study still had clinical significance: (1) Insufficient drug application may lead to high-level resistance of MCZ in future clinical treatment; (2) C387S mutation in dprE1 can be used as a marker for identifying high-level resistant 
strains.

In conclusion, we confirmed that $\mathrm{C} 387 \mathrm{~S}$ mutation in dprE1 is directly related to the emergence of high-level MCZ-resistant strains which can be used as a molecular marker to detect MCZ resistance in future clinic application and identified that there are other drug resistance mechanisms, which are related to low-level resistance. The mechanism of low-level resistance can be clarified by genome sequencing of more kinds of induced strains in future work.

\section{ATERIAS AND METHODS}

Bacterial strains and growth conditions. M.tb H37Rv (ATCC 27294) is frozen in the department of pharmacology of Beijing tuberculosis chest tumor research institute. Subcultures in $7 \mathrm{H} 9$ broth were grown up to the logarithmic phase and bacterial suspension was filtered by $8 \mu \mathrm{m}$ filter membrane to obtain bacterium suspension. Single bacterial suspension was inoculated into 7H10 culture plate after being diluted ten-fold, colony forming unit (CFU) of the single bacterium suspension is determined after 4 weeks. Viable bacterial were preserved in $1.0 \mathrm{ml}$ aliquots frozen at $-80^{\circ} \mathrm{C}$. For each experiment, one aliquot was diluted in $7 \mathrm{H} 9$ broth to obtain a desirable concentration of bacteria.

Susceptibility testing. MCZ susceptibility testing was carried out using MABA method to determine minimum inhibitory concentration ( $\mathrm{MIC}_{90}$ ) as previously described (41). Briefly, prepare M.tb single colony suspension: M.tb is inoculated into 7H9 broth containing $10 \% \mathrm{OADC}$ and $0.05 \%$ tween-80, cultured at $37{ }^{\circ} \mathrm{C}$ and $5 \%$ $\mathrm{CO} 2$ for $2 \sim 3$ weeks to logarithmic growth phase. The isolate in the log phase was diluted to $1 \times 10^{6} \mathrm{cfu} / \mathrm{ml}$ in $7 \mathrm{H} 9$ broth containing $10 \%$ OADC. MCZ was serially diluted twofold in $100 \mu 1$ 7H9 broth, and the final concentrations were $0.5,0.25,0.125$, 
$0.0625, \quad 0.03125,0.016,0.008,0.004,0.002,0.001,0.0005,0.00025$ and

252 $0.000125 \mu \mathrm{g} / \mathrm{ml}$. Then, $100 \mu \mathrm{l}$ bacterial suspension was inoculated to each well of microplates. The final volume of each well was $200 \mu$, containing $100 \mu \mathrm{l}$ of $\mathrm{MCZ}$ solution and $100 \mu \mathrm{l}$ of bacterial suspension. Furthermore, MCZ -free wells were used as positive control to determine the time for adding alamarBlue. Microplates were incubated at $37{ }^{\circ} \mathrm{C}$ for 7 days. $20 \mu 1$ alamarBlue mixed with $12.5 \mu 110 \%$ Tween-80 was added to the Microplates. The minimum inhibitory concentration (MIC90) value was defined as the lowest MCZ concentration that inhibited bacterial growth and prevented a color change.

Protocol for MCZ induction. The method of in vitro induced resistant to MCZ is as shown in figure 1. Briefly, MCZ was dissolved in DMSO at a stock concentration of $1 \mathrm{mg} / \mathrm{mL}$ and incorporated into $7 \mathrm{H} 10$ agar plates containing OADC at concentrations of $0.1 \mathrm{ng} / \mathrm{mL} .5 \times 10^{5} \mathrm{cfu} / \mathrm{ml} \mathrm{H} 37 \mathrm{Rv}$ were inoculated on the above 7H10 agar plate. Isolates that grew on the MCZ containing plates after $3 \sim 4$ weeks incubation at $37^{\circ} \mathrm{C}$ were picked and inoculated on the $7 \mathrm{H} 10$ agar plates containing OADC at concentrations of $0.2 \mathrm{ng} / \mathrm{mL}$. By repeating above steps isolates were obtained from $7 \mathrm{H} 10$ agar plates containing MCZ from $0.1 \mathrm{ng} / \mathrm{mL}-100 \mathrm{ng} / \mathrm{mL}$. All the single colonies were collected and stored in $7 \mathrm{H} 9$ broth at $-80^{\circ} \mathrm{C}$ respectively. Then isolates were grown in $7 \mathrm{H} 9$ broth for confirming $\mathrm{MCZ}$ resistance phenotype by MABA method. Wild type H37Rv was included as a drug susceptible control strain for the MCZ susceptibility testing. In the MCZ susceptibility testing isoniazid (INH) and rifampicin (RIF) was used as control drug. 
from 7H10 agar plates with MCZ concentration of $3.2 \mathrm{ng} / \mathrm{ml} \sim 100 \mathrm{ng} / \mathrm{ml}$ were selected. Genomic DNA was extracted using the Wizard ${ }^{\circledR}$ Genomic DNA Purification Kit (Promega, American) according to the manufacturer's instructions.

resistance. Strains obtained from the $7 \mathrm{H} 10$ agar with $\mathrm{MCZ}$ concentration of $6.4 \mathrm{ng} / \mathrm{ml}$ were selected to carry out PCR sequencing analysis on dprE1 gene. The dprE1 PCR was performed using forword primer (5'-CTCACGCAGTTCTACCATCCG-3') and reverse primer (5'-TCCAGGGCGTCAAAGTCG-3') as described. Briefly, genomic DNA from MCZ-induced strains was used as templates for PCR as follows: heat denaturation at $94{ }^{\circ} \mathrm{C} 5 \mathrm{~min}$ followed by 30 cycles of $94{ }^{\circ} \mathrm{C} 1 \mathrm{~min}, 55{ }^{\circ} \mathrm{C} 1 \mathrm{~min}, 72{ }^{\circ} \mathrm{C}$ $1 \mathrm{~min}$ followed by extension at $72{ }^{\circ} \mathrm{C}$ for $10 \mathrm{~min}$. The PCR reaction was then cooled to $4^{\circ} \mathrm{C}$. The dprE1 PCR products were then sequenced by ABI 377 DNA sequencer at Beijing ReboXingke Biotechnology Co., Ltd., and the dprE1 sequences from different isolates were compared against the wild type dprE1 sequence of H37Rv to identify potential mutations in the dprE1 gene.

Whole genome sequencing. The genomic DNA for whole genome sequencing was isolated as previously described. Sequencing libraries were prepared using the Nextera XT Sample Prep Kit (Illumina, San Diego, CA, USA) according to the manufacturer's protocol and sequenced on Illumina Hiseq and BGISEQ-500 platforms. The sequencing depths is 50-fold coverage. High-quality reads were aligned to the M.tb H37Rv (GenBank NC000962.3) reference sequence using 295 Bowtie2. Single nucleotide polymorphisms (SNPs) and InDels (insertion and deletion) were detected using SAMtools. In order to eliminate the genomic differences of MCZ-resistant islolates and H37Rv in our analysis, SNPs and InDels were further 
299 Synonymous mutations and PE/PPE mutations within coding sequence were removed 300 in the final analysis to focus on mutations that are most likely involved in 301 MCZ-resistance. The whole genome sequencing analysis was carried out in Shanghai 302 Bo-hao Biotechnology Company.

\section{ACKNOWLEDGEMENTS}

305 This work was supported by National Science and Technology Major Project of decision to submit the work for publication.

We have no conflict of interest to declare.

\section{REFERENCES}

1. World Health Organization (WHO), Global Tuberculosis Report 2019. World Health Organization, Geneva, Switzerland.

2. Brouqui P, Quenard F, Drancourt M. 2017. Old antibiotics for emerging multidrug-resistant/extensively drug-resistant tuberculosis (MDR/XDR-TB). International journal

3. Diel R, Vandeputte J, de Vries G, Stillo J, Wanlin M, Nienhaus A. 2014. Costs of tuberculosis

5. Quenard F, Fournier PE, Drancourt M, Brouqui P. 2017. Role of second-line injectable antituberculosis drugs in the treatment of MDR/XDR tuberculosis. International journal of antimicrobial agents 50:252-254. https://doi.org/10.1016/j.jjantimicag.2017.01.042.

6. Ahmad Khan F, Salim MAH, du Cros P, Casas EC, Khamraev A, Sikhondze W, Benedetti A, 
Bastos M, Lan Z, Jaramillo E, Falzon D, Menzies D. 2017. Effectiveness and safety of standardised shorter regimens for multidrug-resistant tuberculosis: individual patient data and aggregate data meta-analyses. Eur Respir J 50:1700061. https://doi.org/10.1183/13993003.00061-2017.

7. Dheda K, Limberis JD, Pietersen E, Phelan J, Esmail A, Lesosky M, Fennelly KP, Te Riele J, Mastrapa B, Streicher EM, Dolby T, Abdallah AM, Ben-Rached F, Simpson J, Smith L, Gumbo T, van Helden P, Sirgel FA, McNerney R, Theron G, Pain A, Clark TG, Warren RM. 2017. Outcomes, infectiousness, and transmission dynamics of patients with extensively drug-resistant tuberculosis and home-discharged patients with programmatically incurable tuberculosis: a prospective cohort study. Lancet Respir Med 5:269-281. http://researchonline.lshtm.ac.uk/3415772/.

8. Bahuguna A, Rawat DS. 2020. An overview of new antitubercular drugs, drug candidates, and their targets. Med Res Rev 40:263-292. https://doi.org/10.1002/med.21602.

9. Tiberi S, du Plessis N, Walzl G, Vjecha MJ, Rao M, Ntoumi F, Mfinanga S, Kapata N, Mwaba P, McHugh TD, Ippolito G, Migliori GB, Maeurer MJ, Zumla A. 2018. Tuberculosis: progress and advances in development of new drugs, treatment regimens, and host-directed therapies. Lancet Infect Dis 18:e183-e198. https://doi.org/10.1016/S1473-3099(18)30110-5.

10. Piton J, Vocat A, Lupien A, Foo CS, Riabova O, Makarov V, Cole ST. 2018. Structure-Based Drug Design and Characterization of Sulfonyl-Piperazine Benzothiazinone Inhibitors of DprE1 from Mycobacterium tuberculosis. Antimicrob Agents Chemother 62:e0681-18. https://doi.org/10.1128/AAC.00681-18.

11. Makarov V, Neres J, Hartkoorn RC, Ryabova OB, Kazakova E, Šarkan M, Huszár S, Piton J, Kolly GS, Vocat A, Conroy TM, Mikušová K, Cole ST. 2015. The 8-Pyrrole-Benzothiazinones Are Noncovalent Inhibitors of DprE1 from Mycobacterium tuberculosis. Antimicrob Agents Chemother 59:4446-4452. https://doi.org/10.1128/AAC.00778-15.

12. Makarov V, Lechartier B, Zhang M, Neres J, van der Sar AM, Raadsen SA, Hartkoorn RC, Ryabova OB, Vocat A, Decosterd LA, Widmer N, Buclin T, Bitter W, Andries K, Pojer F, Dyson PJ, Cole ST. 2014. Towards a new combination therapy for tuberculosis with next generation benzothiazinones. EMBO Mol Med 6:372-383. https://doi.org/10.1002/emmm.201303575.

13. Makarov V, Manina G, Mikusova K, Möllmann U, Ryabova O, Saint-Joanis B, Dhar N, Pasca MR, Buroni S, Lucarelli AP, Milano A, De Rossi E, Belanova M, Bobovska A, Dianiskova P, 
Kordulakova J, Sala C, Fullam E, Schneider P, McKinney JD, Brodin P, Christophe T, Waddell S, Butcher P, Albrethsen J, Rosenkrands I, Brosch R, Nandi V, Bharath S, Gaonkar S, Shandil RK, Balasubramanian V, Balganesh T, Tyagi S, Grosset J, Riccardi G, Cole ST. 2009. Benzothiazinones kill Mycobacterium tuberculosis by blocking arabinan synthesis. Science 324:801-804. https://doi.org/10.1126/science.1171583.

14. Trefzer C, Rengifo-Gonzalez M, Hinner MJ, Schneider P, Makarov V, Cole ST, Johnsson K. 2010. Benzothiazinones: prodrugs that covalently modify the decaprenylphosphoryl- $\beta$-D-ribose 2'-epimerase DprE1 of Mycobacterium tuberculosis. J Am Chem Soc 132:13663-13665. https://doi.org/10.1021/ja106357w.

15. Neres J, Pojer F, Molteni E, Chiarelli LR, Dhar N, Boy-Röttger S, Buroni S, Fullam E, Degiacomi G, Lucarelli AP, Read RJ, Zanoni G, Edmondson DE, De Rossi E, Pasca MR, McKinney JD, Dyson PJ, Riccardi G, Mattevi A, Cole ST, Binda C. 2012. Structural basis for benzothiazinone-mediated killing of Mycobacterium tuberculosis. Sci Transl Med 4:150ra121. https://doi.org/10.1126/scitranslmed.3004395.

16. Wolucka BA. 2008. Biosynthesis of D-arabinose in mycobacteria - a novel bacterial pathway with implications for antimycobacterial therapy. FEBS J 275:2691-2711. https://doi.org/10.1111/j.1742-4658.2008.06395.x.

17. Batt SM, Jabeen T, Bhowruth V, Quill L, Lund PA, Eggeling L, Alderwick LJ, Fütterer K, Besra GS. 2012. Structural basis of inhibition of Mycobacterium tuberculosis DprE1 by benzothiazinone inhibitors. Proc Natl Acad Sci U S A 109:11354-11359. https://doi.org/10.1073/pnas.1205735109.

18. Foo CS, Lechartier B, Kolly GS, Boy-Röttger S, Neres J, Rybniker J, Lupien A, Sala C, Piton J, Cole ST. 2016. Characterization of DprE1-Mediated Benzothiazinone Resistance in Mycobacterium tuberculosis. Antimicrob Agents Chemother 60:6451-6459. https://doi.org/10.1128/AAC.01523-16.

19. Shi J, Lu J, Wen S, Zong Z, Huo F, Luo J, Liang Q, Li Y, Huang H, Pang Y. 2018. In Vitro Activity of PBTZ169 against Multiple Mycobacterium Species. Antimicrob Agents Chemother 62:e01314-18. https://doi.org/10.1128/AAC.01314-18.

20. Manina G, Pasca MR, Buroni S, De Rossi E, Riccardi G. 2010. Decaprenylphosphoryl- $\beta$-D-ribose 2'-epimerase from Mycobacterium tuberculosis is a magic drug target. Curr Med Chem 17:3099-3108. https://doi.org/10.2174/092986710791959693. 
21. Drobniewski F, Rüsch-Gerdes S, Hoffner S; Subcommittee on Antimicrobial Susceptibility Testing of Mycobacterium tuberculosis of the European Committee for Antimicrobial Susceptibility Testing (EUCAST) of the European Society of Clinical Microbiology and Infectious Diseases (ESCMID). 2007. Antimicrobial susceptibility testing of Mycobacterium tuberculosis (EUCAST document E.DEF 8.1)--report of the Subcommittee on Antimicrobial Susceptibility Testing of Mycobacterium tuberculosis of the European Committee for Antimicrobial Susceptibility Testing (EUCAST) of the European Society of Clinical Microbiology and Infectious Diseases (ESCMID). Clin Microbiol Infect 13:1144-56. https://doi.org/10.1111/j.1469-0691.2007.01813.x.

22. Medical Research Council. 1962. Long-term chemotherapy in the treatment of chronic pulmonary tuberculosis with cavitation. Tubercle 43: 201-67.

23. Crofton J, Mitchison DA. 1948. Streptomycin resistance in pulmonary tuberculosis. Br Med J 2: $1009-15$.

24. David HL. 1970. Probability distribution of drug-resistant mutants in unselected populations of Mycobacterium tuberculosis. Appl Microbiol 20: 810-4.

25. Björkman J, Hughes D, Andersson DI. 1998. Virulence of antibiotic-resistant Salmonella typhimurium. Proc Natl Acad Sci U S A 95: 3949-3953. https://doi.org/10.1073/pnas.95.7.3949.

26. Moura de Sousa J, Balbontín R, Durão P, Gordo I. 2017. Multidrug-resistant bacteria compensate for the epistasis between resistances. PLoS Biol 15:e2001741. https://doi.org/10.1371/journal.pbio.2001741.

27. Qi Q, Preston GM, MacLean RC. 2014. Linking system-wide impacts of RNA polymerase mutations to the fitness cost of rifampin resistance in Pseudomonas aeruginosa. mBio 5:e01562. https://doi.org/10.1128/mBio.01562-14.

28. Nielsen KL, Pedersen TM, Udekwu KI, Petersen A, Skov RL, Hansen LH, Hughes D, Frimodt-Møller N. 2012. Fitness cost: a bacteriological explanation for the demise of the first international methicillin-resistant Staphylococcus aureus epidemic. J Antimicrob Chemother 67:1325-32. https://doi.org/10.1093/jac/dks051.

29. Luo T, Yuan J, Peng X, Yang G, Mi Y, Sun C, Wang C, Zhang C, Bao L. 2017. Double mutation in DNA gyrase confers moxifloxacin resistance and decreased fitness of Mycobacterium smegmatis. J Antimicrob Chemother 72:1893-1900. https://doi.org/10.1093/jac/dkx110. 
30. Melnyk AH, McCloskey N, Hinz AJ, Dettman J, Kassen R. 2017. Evolution of Cost-Free Resistance under Fluctuating Drug Selection in Pseudomonas aeruginosa. mSphere. 2:e00158-17. https://doi.org/10.1128/mSphere.00158-17.

31. Melnyk AH, Wong A, Kassen R. 2015. The fitness costs of antibiotic resistance mutations. Evol Appl 8:273-83. https://doi.org/10.1111/eva.12196.

32. Koch A, Mizrahi V, Warner DF. 2014. The impact of drug resistance on Mycobacterium tuberculosis physiology: what can we learn from rifampicin? Emerg Microbes Infect 3:e17. https://doi.org/10.1038/emi.2014.17.

33. Li Z, Kelley C, Collins F, Rouse D, Morris S. 1998. Expression of katG in Mycobacterium tuberculosis is associated with its growth and persistence in mice and guinea pigs. $\mathrm{J}$ Infect Dis 177:1030-1035. https://doi.org/10.1086/515254.

34. Mariam DH, Mengistu Y, Hoffner SE, Andersson DI. 2004. Effect of rpoB mutations conferring rifampin resistance on fitness of Mycobacterium tuberculosis. Antimicrob Agents Chemother 48:1289-1294. https://doi.org/10.1128/aac.48.4.1289-1294.2004.

35. Gagneux S, Long CD, Small PM, Van T, Schoolnik GK, Bohannan BJ. 2006. The competitive cost of antibiotic resistance in Mycobacterium tuberculosis. Science 312:1944-1946. https://doi.org/10.1126/science.1124410.

36. Sherman DR, Mdluli K, Hickey MJ, Arain TM, Morris SL, Barry CE 3rd, Stover CK. 1996. Compensatory ahpC gene expression in isoniazid-resistant Mycobacterium tuberculosis. Science 272:1641-1643. https://doi.org/10.1126/science.272.5268.1641.

37. Heym B, Stavropoulos E, Honoré N, Domenech P, Saint-Joanis B, Wilson TM, Collins DM, Colston MJ, Cole ST. 1997. Effects of overexpression of the alkyl hydroperoxide reductase AhpC on the virulence and isoniazid resistance of Mycobacterium tuberculosis. Infect Immun 65:1395-1401. https://doi.org/10.1128/IAI.65.4.1395-1401.1997.

38. Comas I, Borrell S, Roetzer A, Rose G, Malla B, Kato-Maeda M, Galagan J, Niemann S, Gagneux S. 2011. Whole-genome sequencing of rifampicin-resistant Mycobacterium tuberculosis strains identifies compensatory mutations in RNA polymerase genes. Nat Genet 44:106-10. https://doi.org/10.1038/ng.1038.

39. de Vos M, Müller B, Borrell S, Black PA, van Helden PD, Warren RM, Gagneux S, Victor TC. 2013. Putative compensatory mutations in the rpoC gene of rifampin-resistant Mycobacterium 
tuberculosis are associated with ongoing transmission. Antimicrob Agents Chemother 57:827-832. https://doi.org/10.1128/AAC.01541-12.

40. Springer B, Master S, Sander P, Zahrt T, McFalone M, Song J, Papavinasasundaram KG, Colston MJ, Boettger E, Deretic V. 2001. Silencing of oxidative stress response in Mycobacterium tuberculosis: expression patterns of ahpC in virulent and avirulent strains and effect of ahpC inactivation. Infect Immun 69:5967-5973. https://doi.org/10.1128/IAI.69.10.5967-5973.2001. 2008. Inter- and intra-assay reproducibility of microplate Alamar blue assay results for isoniazid, rifampicin, ethambutol, streptomycin, ciprofloxacin, and capreomycin drug susceptibility testing of Mycobacterium tuberculosis. J Clin Microbiol 46:3526-3529. 
Figure 1 The method of in vitro induced resistant to MCZ.

H37Rv Parent culture

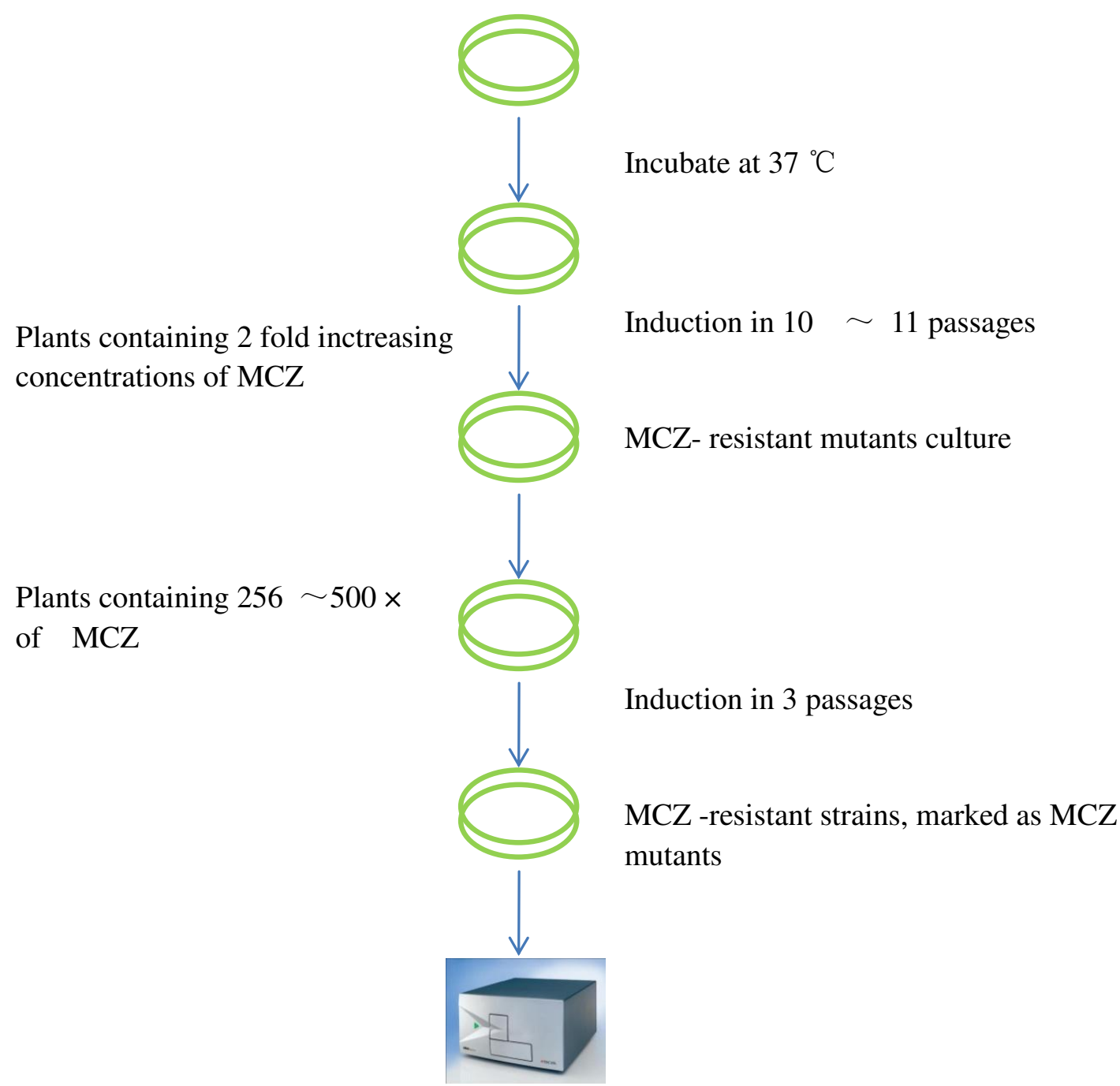

Phenotypic confirmation: MIC determination 
1 Table 1 MCZ sensitivity detection of induced strains ( $\mathrm{MIC}_{90}, \mu \mathrm{g} / \mathrm{ml}$ )

\begin{tabular}{|c|c|c|c|c|c|c|c|c|c|c|c|}
\hline \multicolumn{2}{|c|}{$0.0032 \mu \mathrm{g} / \mathrm{ml}$} & \multicolumn{2}{|c|}{$0.0064 \mu \mathrm{g} / \mathrm{ml}$} & \multicolumn{2}{|c|}{$0.0064 \mathrm{\mu g} / \mathrm{ml} \mathrm{I}$} & \multicolumn{2}{|c|}{$0.0256 \mu \mathrm{g} / \mathrm{ml} \mathrm{I}$} & \multicolumn{2}{|c|}{$0.05 \mu \mathrm{g} / \mathrm{ml}$} & \multicolumn{2}{|c|}{$0.1 \mu \mathrm{g} / \mathrm{ml}$} \\
\hline Strain number & $\mathrm{MIC}_{90}$ & Strain number & $\mathrm{MIC}_{90}$ & Strain number & $\mathrm{MIC}_{90}$ & Strain number & $\mathrm{MIC}_{90}$ & Strain number & $\mathrm{MIC}_{90}$ & Strain number & $\mathrm{MIC}_{90}$ \\
\hline \multirow[t]{10}{*}{$4-5$} & 0.0010 & $1-4$ & 0.0053 & $26-4$ & 0.0046 & $1-3$ & 0.0070 & $1-2$ & 0.0068 & $1-1$ & $>0.5$ \\
\hline & & $2-4$ & 0.0059 & $27-4$ & 0.0039 & $2-3$ & 0.0060 & $2-2$ & 0.0053 & $2-1$ & $>0.5$ \\
\hline & & $3-4$ & 0.0055 & $28-4$ & 0.0061 & $3-3$ & 0.0028 & $3-2$ & 0.0027 & $3-1$ & $>0.5$ \\
\hline & & $4-4$ & $>0.5$ & $29-4$ & 0.0103 & $4-3$ & $>0.5$ & $4-2$ & $>05$ & $4-1$ & $>0.5$ \\
\hline & & $5-4$ & 0.0030 & $30-4$ & 0.0036 & $5-3$ & 0.0045 & $5-2$ & 0.0036 & & \\
\hline & & $6-4$ & 0.0048 & $31-4$ & 0.0043 & $6-3$ & 0.0117 & $6-2$ & 0.0077 & & \\
\hline & & $7-4$ & 0.0038 & $32-4$ & 0.0038 & $7-3$ & 0.0076 & $7-2$ & 0.0091 & & \\
\hline & & $8-4$ & 0.0031 & $33-4$ & 0.0031 & $8-3$ & 0.0058 & $8-2$ & 0.0053 & & \\
\hline & & $9-4$ & 0.0068 & $34-4$ & 0.0020 & $9-3$ & 0.0094 & $9-2$ & 0.0101 & & \\
\hline & & $10-4$ & 0.0048 & $35-4$ & 0.0036 & $10-3$ & 0.0050 & $10-2$ & 0.0035 & & \\
\hline
\end{tabular}




\begin{tabular}{|c|c|c|c|c|c|c|c|}
\hline $11-4$ & 0.0031 & $36-4$ & 0.0031 & $11-3$ & 0.0044 & $11-2$ & 0.0064 \\
\hline $12-4$ & 0.0030 & $37-4$ & 0.0034 & $12-3$ & 0.0021 & $12-2$ & 0.0048 \\
\hline $13-4$ & 0.0044 & $38-4$ & 0.0034 & $13-3$ & 0.0115 & $13-2$ & 0.0113 \\
\hline $14-4$ & 0.0053 & $39-4$ & 0.0030 & $14-3$ & 0.0080 & $14-2$ & 0.0093 \\
\hline $15-4$ & 0.0048 & $40-4$ & 0.0048 & $15-3$ & 0.0050 & $15-2$ & 0.0060 \\
\hline $16-4$ & 0.0030 & $41-4$ & 0.0034 & $16-3$ & 0.0030 & $16-2$ & 0.0040 \\
\hline $17-4$ & 0.0034 & $42-4$ & 0.0032 & $17-3$ & 0.0054 & $17-2$ & 0.0055 \\
\hline $18-4$ & 0.0030 & $43-4$ & 0.0029 & $18-3$ & 0.0031 & $18-2$ & 0.0042 \\
\hline $19-4$ & 0.0032 & $44-4$ & 0.0027 & $19-3$ & 0.0057 & $19-2$ & 0.0041 \\
\hline 20-4 & 0.0051 & $45-4$ & 0.0030 & $20-3$ & 0.0058 & $20-2$ & 0.0120 \\
\hline $21-4$ & 0.0037 & $46-4$ & 0.0038 & & & & \\
\hline $22-4$ & 0.0031 & $47-4$ & 0.0039 & & & & \\
\hline $23-4$ & 0.0045 & $48-4$ & 0.0033 & & & & \\
\hline
\end{tabular}


3 concentrations, and the rest are the same. The content in brackets indicates the MCZ concentration in the culture medium. 
1 Table 2 Genome sequencing of some strains

\begin{tabular}{|c|c|}
\hline Strain number & genome sequencing \\
\hline $2-1$ & dprE1(C387S), rv0678(A99V),rrs(Q1026H),mbtF(A270G),rv2956(A108T),dprE1(G248A),fadD2(R137P),phoT ( Q153R ),rv1725c (E28G),SenX3 (frameshift) \\
\hline $2-2$ & rv0678(A99V),rrs(Q1026H),mbtF(A270G),rv2956(A108T),dprE1(G248A),fadD2(R137P), phoT(Q153R),rv1725c (E28G),SenX3 (frameshift) \\
\hline $2-4$ & rv0678(A99V),rrs(Q1026H),mbtF(A270G),rv2956(A108T),dprE1(G248A),fadD2(R137P), phoT(Q153R),rv1725c (E28G),SenX3 (frameshift) \\
\hline 3-1 & dprE1(C387S),dprE1(G61A),fadD2(R137P), phoT (Q153R),rv1725c(E28G), ,SenX3 (frameshift), rv3230c(S290P),intergenic region \\
\hline $3-2$ & intergenicregion,dprE1 (G61A),fadD2(R137P),phoT(Q153R),rv1725c(E28G),SenX3 (frameshift) \\
\hline 3-4 & dprE1 (G61A),fadD2 (R137P), phoT ( Q153R ), rv1725c (E28G),SenX3 (frameshift) \\
\hline $4-1$ & dprE1(C387S), mbtF(A270G), aofH(G383S),fadD2(R137P),phoT (Q153R), rv1725c (E28G),SenX3 (frameshift) \\
\hline 4-4 & dprE1(C387S), mbtF(A270G),aofH(G383S),fadD2(R137P), phoT (Q153R), rv1725c (E28G) ,SenX3 (frameshift) \\
\hline 4-5 & mbtF(A270G),fadD2(R137P), phoT (Q153R), rv1725c (E28G),SenX3 (frameshift) \\
\hline
\end{tabular}

2 Note: Description of strain number: 1-1, 1-2, 1-3 and 1-4 represent the corresponding strains from the same source induced by different drug

3 concentrations, and the rest are the same. 